\title{
Hybrid Robust Optimization for the Design of a Smartphone Metal Frame Antenna
}

\author{
Sungwoo Lee, ${ }^{1}$ Jong Min Lee, ${ }^{1}$ Keum Cheol Hwang $\mathbb{D}^{1},{ }^{1}$ Sanguk Park, ${ }^{2}$ and Soonyong Lee ${ }^{2}$ \\ ${ }^{1}$ School of Electronic and Electrical Engineering, Sungkyunkwan University, Suwon 440-746, Republic of Korea \\ ${ }^{2}$ Global Technology Center, Samsung Electronics Co. Ltd., Suwon, Republic of Korea \\ Correspondence should be addressed to Keum Cheol Hwang; khwang@skku.edu
}

Received 3 August 2017; Revised 27 November 2017; Accepted 26 December 2017; Published 18 February 2018

Academic Editor: Sotirios K. Goudos

Copyright (C) 2018 Sungwoo Lee et al. This is an open access article distributed under the Creative Commons Attribution License, which permits unrestricted use, distribution, and reproduction in any medium, provided the original work is properly cited.

\begin{abstract}
Hybrid robust optimization that combines a genetical swarm optimization (GSO) scheme with an orthogonal array (OA) is proposed to design an antenna robust to the tolerances arising during the fabrication process of the antenna in this paper. An inverted-F antenna with a metal frame serves as an example to explain the procedure of the proposed method. GSO is adapted to determine the design variables of the antenna, which operates on the GSM850 band $(824-894 \mathrm{MHz})$. The robustness of the antenna is evaluated through a noise test using the OA. The robustness of the optimized antenna is improved by approximately $61.3 \%$ relative to that of a conventional antenna. Conventional and optimized antennas are fabricated and measured to validate the experimental results.
\end{abstract}

\section{Introduction}

Recently, as the interest in smartphones has increased in the mobile communication market, metal frames are becoming widely used to provide the consumer with a stylish and luxurious exterior for their devices. However, when the metal frame is near the antenna in a smartphone, it causes undesired coupling effects which degrade the antenna performance [1]. The performance can also be deteriorated due to the tolerances arising during the manufacturing process of an antenna. Therefore, noise factors such as tolerance levels should be considered in the design of an antenna. Taguchi's method (TM) is a well-known technique which can be used to find a robustly optimized condition against such noise factors [2]. Moreover, a method which is realized on the basis of an orthogonal array $(\mathrm{OA})$ can dramatically reduce the time required to design the antenna [3]. However, it is very difficult to find a global optimum using only TM because an OA consists of a limited number and limited level sizes of design variables [4]. In order to resolve the drawbacks of TM, hybrid methods have been introduced [5-7]. In one study [5], a hybrid method which combined TM with an evolution strategy (ES) was proposed. The ES is used to determine the geometry of the antenna, which operates at around $920 \mathrm{MHz}$. Subsequently, based on the result acquired from the ES, a robust antenna configuration is obtained by TM. TM with a genetic algorithm (GA) or particle swarm optimization (PSO) was also investigated in schemes where robust genes or particles were selected and utilized $[6,7]$.

In the present paper, a hybrid robust optimization (HRO) which combines genetical swarm optimization (GSO) with an OA is proposed. The standard TM is inefficient when used with an extensive search boundary because it considers only limited cases. To overcome this drawback, the GSO algorithm is used instead of OA to obtain a new combination of design parameters in the proposed method. GSO as used here is developed based on PSO, and randomly chosen parts of the population evolve with a GA during each iteration. Accordingly, GSO is less likely to fall to the local optimum and its convergence speed is also faster than those of the GA and PSO methods [8]. Besides, the total number of experiments is reduced because an $\mathrm{OA}$ is used for noise testing. An inverted-F antenna (IFA) with a metal frame [9-11] is employed as an example to explain the procedure of the proposed method. The geometry of the IFA is illustrated in Section 2. The procedure of the HRO is described 


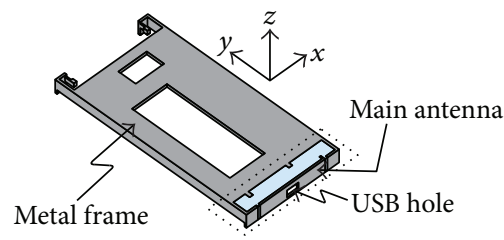

(a)

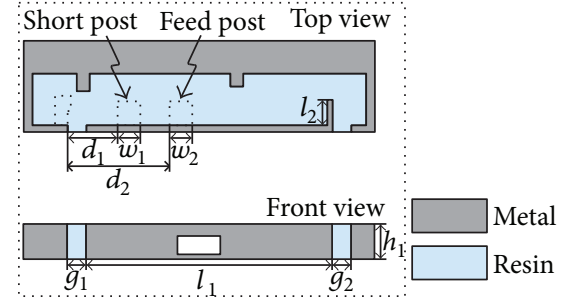

(b)

FIGURE 1: The geometry of a smartphone body and main antenna: (a) 3D view of the smartphone body and (b) expanded view of the main antenna.

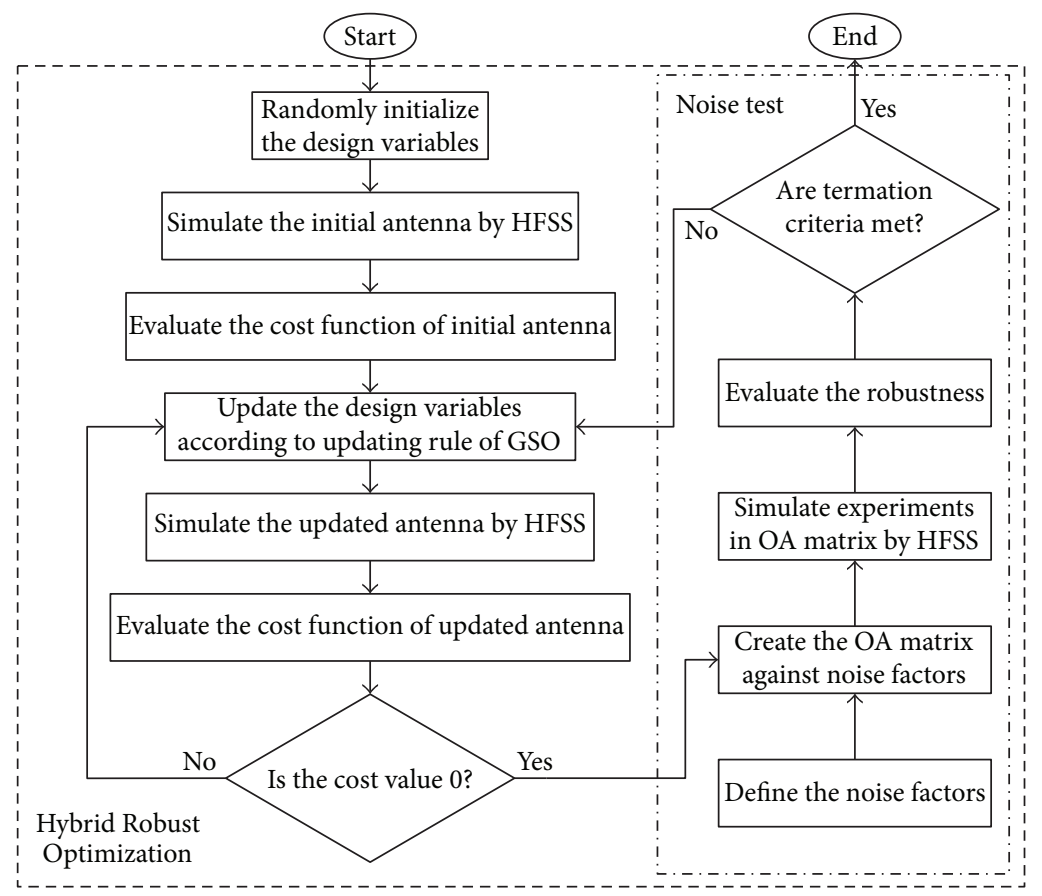

Figure 2: Flow chart of the proposed hybrid robust optimization scheme.

in detail in Section 3. The robust optimization result is depicted in Section 4. The experimental results are demonstrated in Section 5.

\section{Geometry}

In general, the main antenna in a smartphone is positioned at the bottom, as shown in Figure 1(a). Figure 1(b) shows the detailed configuration of the main antenna in this case, which operates as an IFA. The antenna is aligned with the protruding L-shaped and inverted L-shaped metal frame. A rectangular hole in the antenna exists for the universal serial bus connector. $g_{1}$ and $g_{2}$ denote the left and right gaps between the antenna and the metal frame, respectively. The length of the antenna is defined as $l_{1}$, and that of the tail of the antenna is denoted by $l_{2} . w_{1}$ and $w_{2}$ are widths of the short and feed posts, respectively. The distances from the edge of the protruding L-shaped metal frame to the left edge of the short post and to that of the feed post are correspondingly represented by $d_{1}$ and $d_{2} . h_{1}$ is the height of the antenna. The dimensions of the conventional main antenna are as
TABLE 1: Optimization range of design variables.

\begin{tabular}{cccccccccc}
\hline & \multicolumn{10}{c}{ Design variable (mm) } \\
& $g_{1}$ & $g_{2}$ & $l_{1}$ & $l_{2}$ & $w_{1}$ & $w_{2}$ & $d_{1}$ & $d_{2}$ & $h_{1}$ \\
\hline $\operatorname{Max}$ & 0.2 & 0.2 & 47.2 & 0 & 1.5 & 1.5 & 3.7 & 11.5 & 6.9 \\
$\operatorname{Min}$ & 3.5 & 3.5 & 49.2 & 10 & 3.5 & 3.5 & 5.7 & 13.5 & 7.9 \\
\hline
\end{tabular}

follows: $g_{1}=1.7 \mathrm{~mm}, g_{2}=1.7 \mathrm{~mm}, l_{1}=48.2 \mathrm{~mm}, l_{2}=2 \mathrm{~mm}$, $w_{1}=2 \mathrm{~mm}, w_{2}=2 \mathrm{~mm}, d_{1}=4.4 \mathrm{~mm}, d_{2}=13.8 \mathrm{~mm}$, and $h_{1}=6.9 \mathrm{~mm}$.

\section{Hybrid Robust Optimization}

Figure 2 shows a flow chart of the proposed HRO. This method is conducted on the basis of GSO, and the robustness of the antenna is evaluated through the OA matrix. The detailed procedure for the HRO is given below:

(1) Randomly initialize the design variables within the range given in Table 1. 


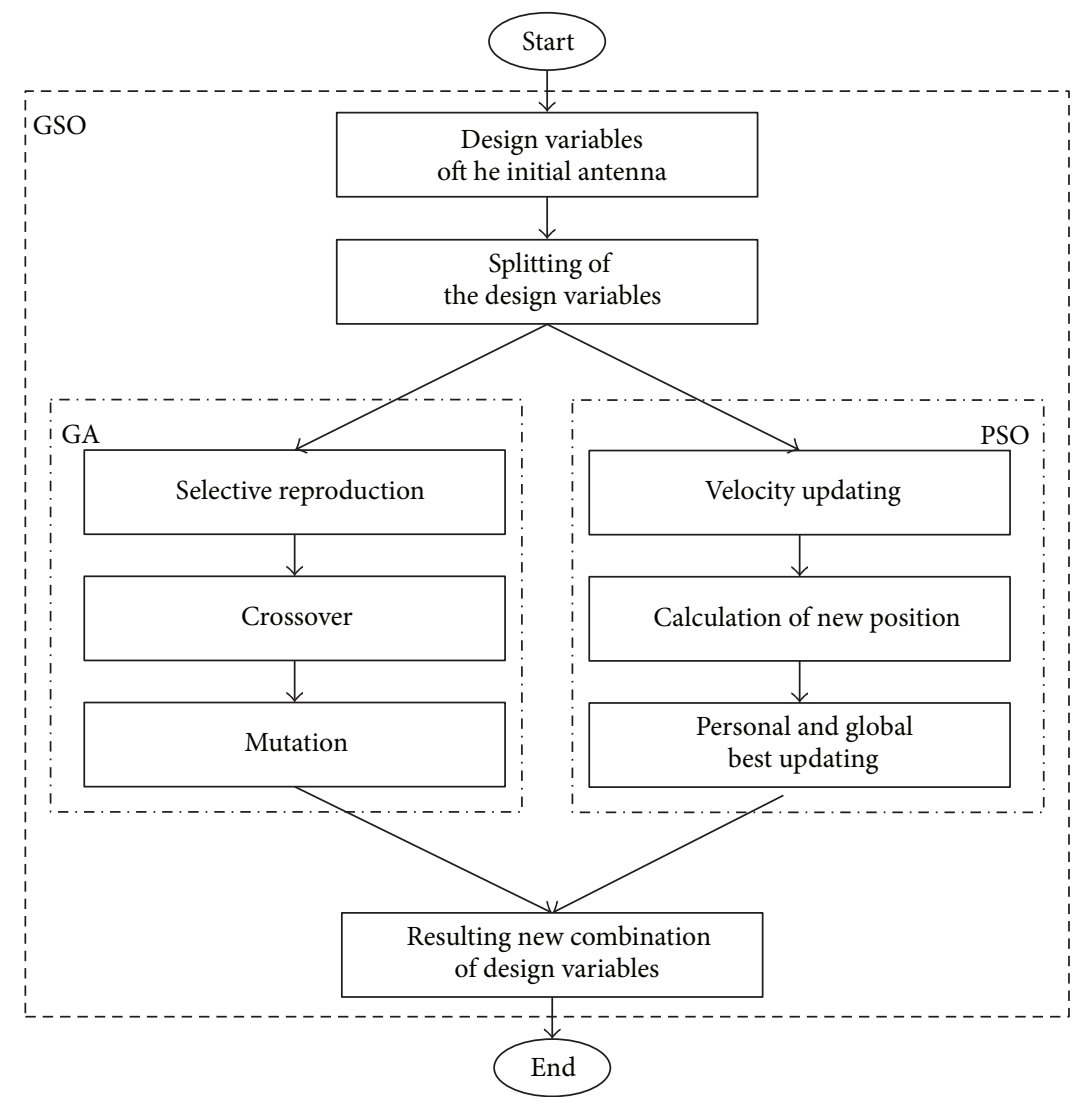

FIgURE 3: Flowchart of the updating rule of GSO.

(2) Simulate the initial antenna using ANSYS highfrequency structure simulation (HFSS) software, and evaluate the cost function of the antenna. The cost function is defined such that the $-6 \mathrm{~dB}$ reflection bandwidth of the antenna covers the GSM850 (824$894 \mathrm{MHz}$ ) band,

$$
\text { cost }=\sum_{i=1}^{n} F(i) \text {, }
$$

where

$$
F(i)= \begin{cases}1, & \text { if } S_{11}\left(f_{i}\right)>-6 \mathrm{~dB}, \\ 0, & \text { otherwise, }\end{cases}
$$

where $f_{i}$ is $i$ th sampling frequency within the GSM850 band, $S_{11}\left(f_{i}\right)$ is reflection coefficient at each sampling frequency, and $n$ is the number of sampling frequencies. In this paper, $n$ is set to 71 .

(3) Update the design variables according to the updating rule of GSO. Figure 3 shows a flowchart of the updating rule of GSO. In this paper, GSO with a hybridization coefficient of 0.2 is realized. Specifically, $20 \%$ of the design variables are optimized by the GA while the others are optimized by the PSO. The optimization performance is usually dependent on the level of the hybridization coefficient. It was found that a hybridization coefficient of 0.2 is optimum in this optimization problem. An analysis of the performance of the GSO algorithm is available in the literature [8]. The GSO converges more rapidly than the GA or PSO under any search condition.

(4) Simulate the updated antenna, and evaluate the cost function of the antenna based on (1).

(5) Steps 3 to 4 are repeated until the cost function is 0.

(6) Define the noise factors, and create the OA matrix against the noise factors [12]. In this article, the noise factor is defined as the parameter that affects the resonant frequency of the conventional main antenna. Figure 4 shows the simulated resonant frequencies of the conventional antenna while changing the design variables by $\pm 0.2 \mathrm{~mm}$. The variances of the resonant frequency of the antenna in accordance with the changes in each design variable are listed in Table 2. Among the nine design variables, the four parameters of $g_{2}, l_{1}, l_{2}$, and $h_{1}$ that have resonant frequency variance of $4 \mathrm{MHz}$ or more are used as the noise factors in this paper.

(7) Create the OA $(9,4,3$, and 0.2) matrix against the noise factors, and implement the experiments in the matrix using HFSS.

(8) Evaluate the robustness of the antenna as optimized by GSO. The robustness is defined as the variance 


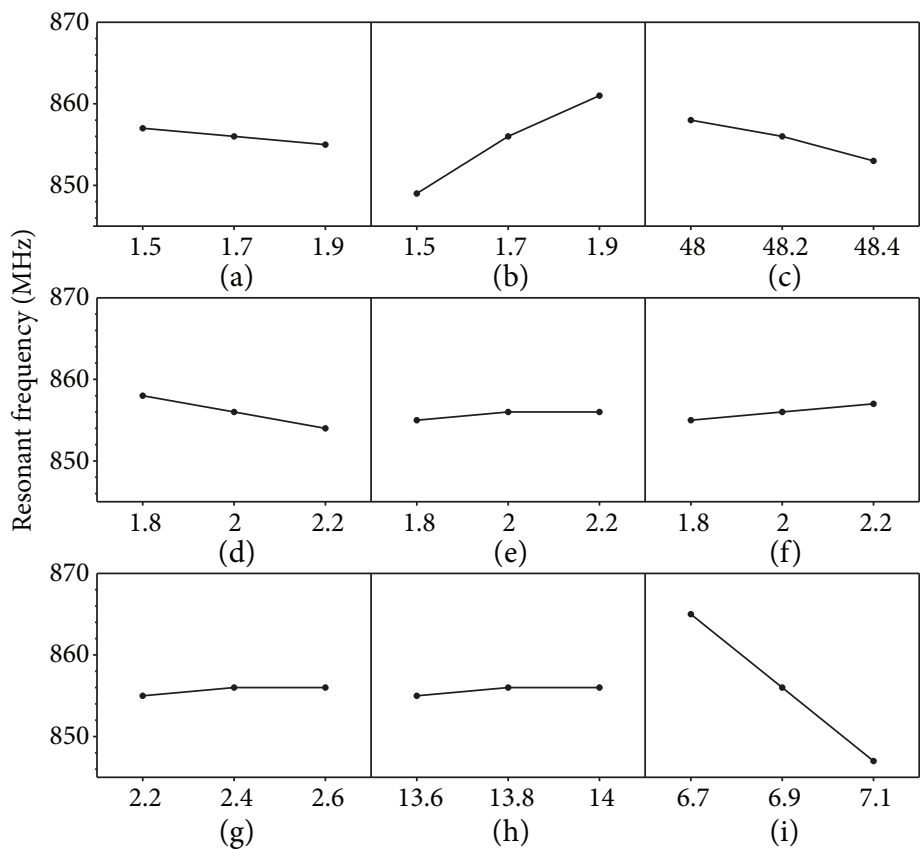

FIGURE 4: Simulated resonant frequencies of the conventional antenna according to the changes in (a) $g_{1}$, (b) $g_{2}$, (c) $l_{1}$, (d) $l_{2}$, (e) $w_{1}$, (f) $w_{2}$, (g) $d_{1}$, (h) $d_{2}$, and (i) $h_{1}$.

TABLE 2: Variance of the resonant frequency of each design variable.

\begin{tabular}{lc}
\hline Design variable & Variance \\
\hline$g_{1}$ & $2 \mathrm{MHz}$ \\
$l 1$ & $5 \mathrm{MHz}$ \\
$w_{1}$ & $1 \mathrm{MHz}$ \\
$d_{1}$ & $1 \mathrm{MHz}$ \\
$h_{1}$ & $18 \mathrm{MHz}$ \\
$g_{2}$ & $12 \mathrm{MHz}$ \\
$l 2$ & $4 \mathrm{MHz}$ \\
$w_{2}$ & $2 \mathrm{MHz}$ \\
$d_{2}$ & $1 \mathrm{MHz}$ \\
\hline
\end{tabular}

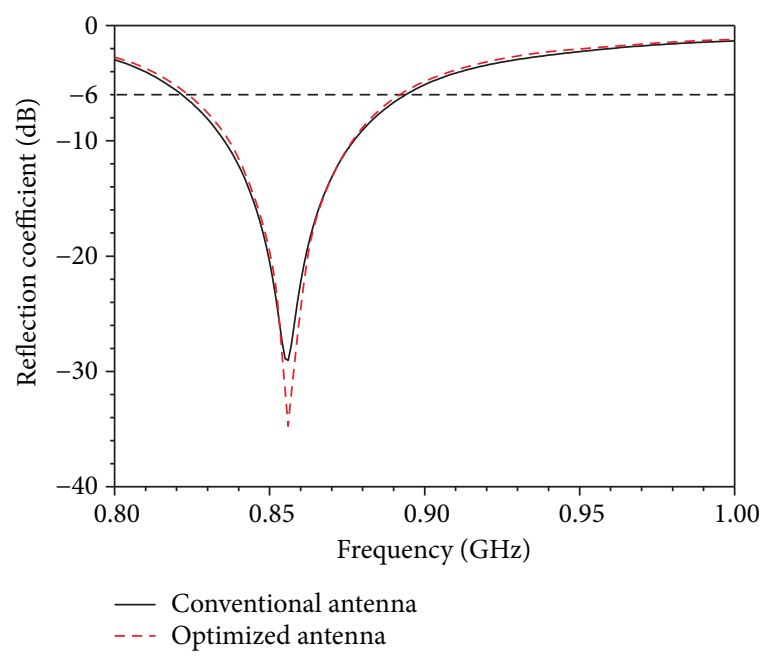

FIgURE 5: Simulated reflection coefficients of the conventional and optimized antennas.

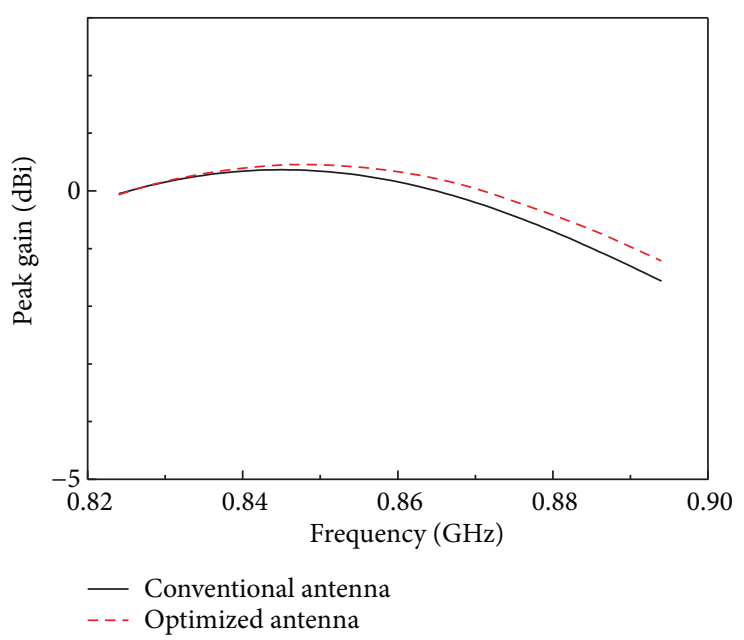

FIGURE 6: Simulated peak gains of the conventional and optimized antennas.

of the resonant frequency obtained from the experiments in the OA matrix:

$$
\text { Robustness }=\mathrm{f}_{\mathrm{r}}(\max )-\mathrm{f}_{\mathrm{r}}(\min ),
$$

where $f_{r}(\max )$ and $f_{r}(\min )$ are the maximum and minimum resonant frequencies in the experiments, respectively.

(9) When the robustness meets the termination criteria, the process of the proposed method is terminated. The termination criteria stipulate that the robustness of the optimized main antenna must be improved by more than $50 \%$ as compared to that of the conventional antenna. 


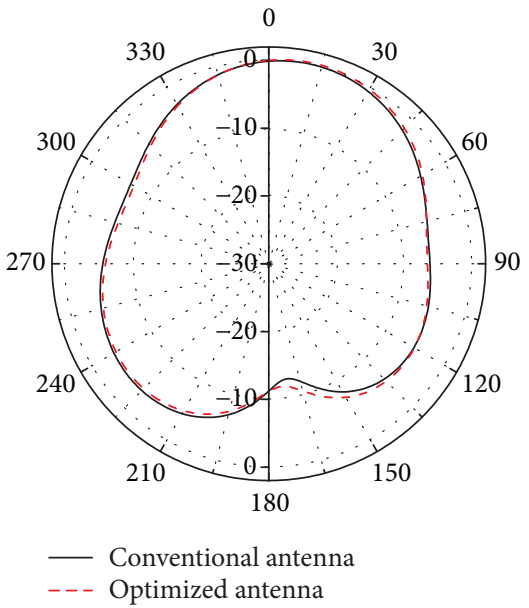

(a)

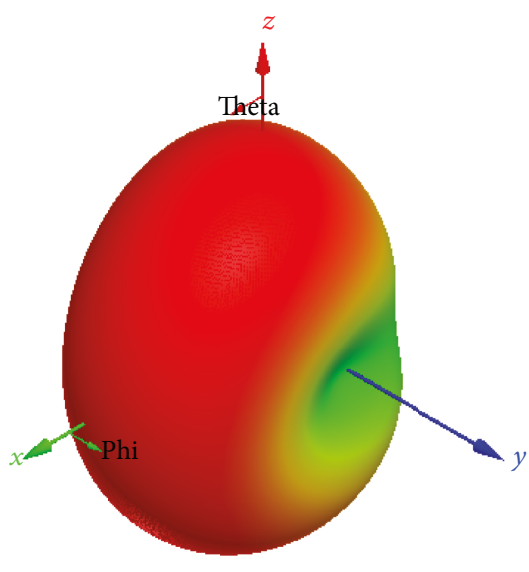

(d)

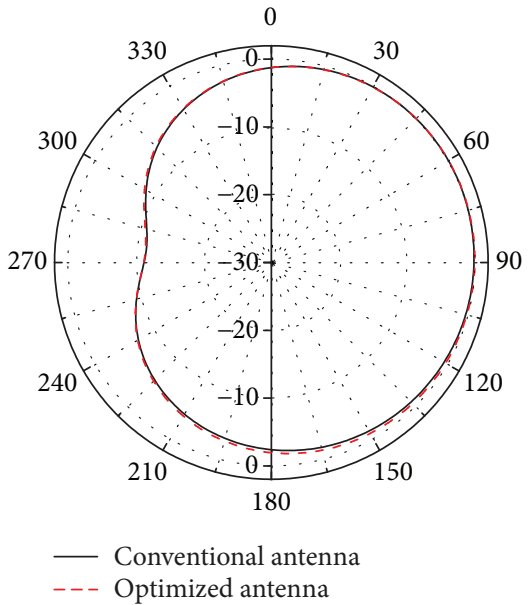

(b)

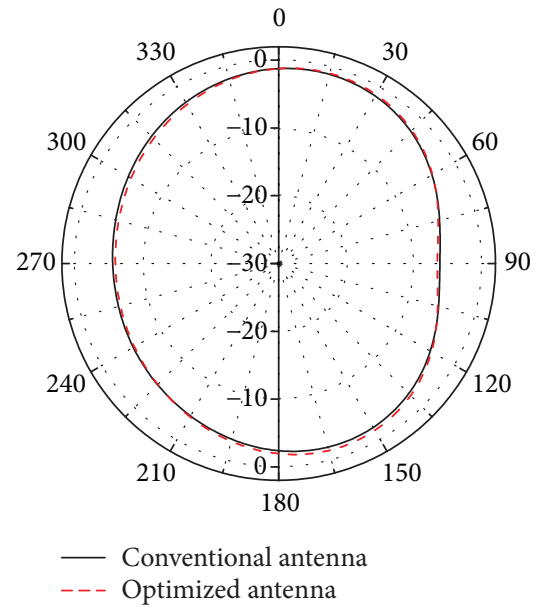

(c)
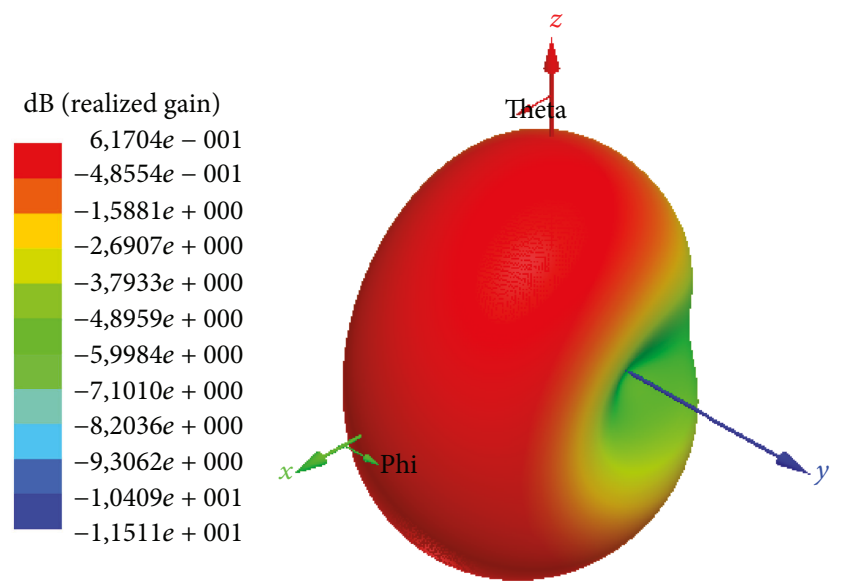

$\mathrm{dB}$ (realized gain)

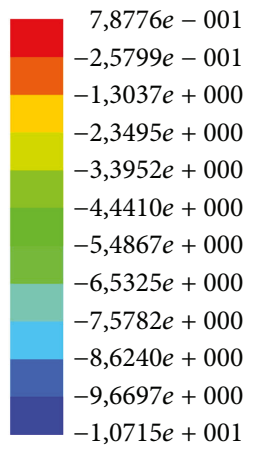

(e)

FIGURE 7: Simulated radiation patterns of the conventional and optimized antennas: (a) $x y$-plane, (b) $x z$-plane, (c) $y z$-plane, (d) 3D radiation pattern of the conventional antenna, and (e) 3D radiation pattern of the optimized antenna.

TABle 3: OA $(9,4,3$, and 0.2) matrix for the conventional antenna.

\begin{tabular}{lcccc}
\hline Experiment & \multicolumn{4}{c}{ Design variable $(\mathrm{mm})$} \\
\hline 1 & $g_{2}$ & $l_{1}$ & $l_{2}$ & $h_{1}$ \\
2 & 1.5 & 48 & 1.8 & 6.7 \\
3 & 1.5 & 48.2 & 2 & 6.9 \\
4 & 1.5 & 48.4 & 2.2 & 7.1 \\
5 & 1.7 & 48 & 2 & 7.1 \\
6 & 1.7 & 48.2 & 2.2 & 6.7 \\
7 & 1.7 & 48.4 & 1.8 & 6.9 \\
8 & 1.9 & 48 & 2.2 & 6.9 \\
9 & 1.9 & 48.2 & 1.8 & 7.1 \\
\hline
\end{tabular}

\section{Robust Optimization Result}

The simulated reflection coefficients of the conventional and optimized antennas are depicted in Figure 5. The $-6 \mathrm{~dB}$ reflection bandwidths of the conventional and optimized antennas are $72 \mathrm{MHz}(822-894 \mathrm{MHz})$ and $70 \mathrm{MHz}$ $(824-894 \mathrm{MHz})$, respectively. Therefore, the optimized
TABle 4: OA $(9,4,3$, and 0.2$)$ matrix for the optimized antenna.

\begin{tabular}{lcccc}
\hline \multirow{2}{*}{ Experiment } & \multicolumn{4}{c}{ Design variable $(\mathrm{mm})$} \\
\hline 1 & $g_{2}$ & $l_{1}$ & $l_{2}$ & $h_{1}$ \\
2 & 2.6 & 48 & 4 & 6.7 \\
3 & 2.6 & 48.2 & 4.2 & 6.9 \\
4 & 2.6 & 48.4 & 4.4 & 7.1 \\
5 & 2.8 & 48 & 4.2 & 7.1 \\
6 & 2.8 & 48.2 & 4.4 & 6.7 \\
7 & 2.8 & 48.4 & 4 & 6.9 \\
8 & 3 & 48 & 4.4 & 6.9 \\
9 & 3 & 48.2 & 4 & 7.1 \\
\hline
\end{tabular}

antenna covers the GSM850 band. The dimensions of the optimized antenna are as follows: $g_{1}=1.7 \mathrm{~mm}, g_{2}=2.8 \mathrm{~mm}$, $l_{1}=48.2 \mathrm{~mm}, l_{2}=4.2 \mathrm{~mm}, w_{1}=2 \mathrm{~mm}, w_{2}=2 \mathrm{~mm}, d_{1}=$ $4.5 \mathrm{~mm}, d_{2}=13.5 \mathrm{~mm}$, and $h_{1}=6.9 \mathrm{~mm}$.

The simulated peak gains of the conventional and optimized antennas are shown in Figure 6. In the GSM850 band, the peak gains of the conventional and optimized antennas 

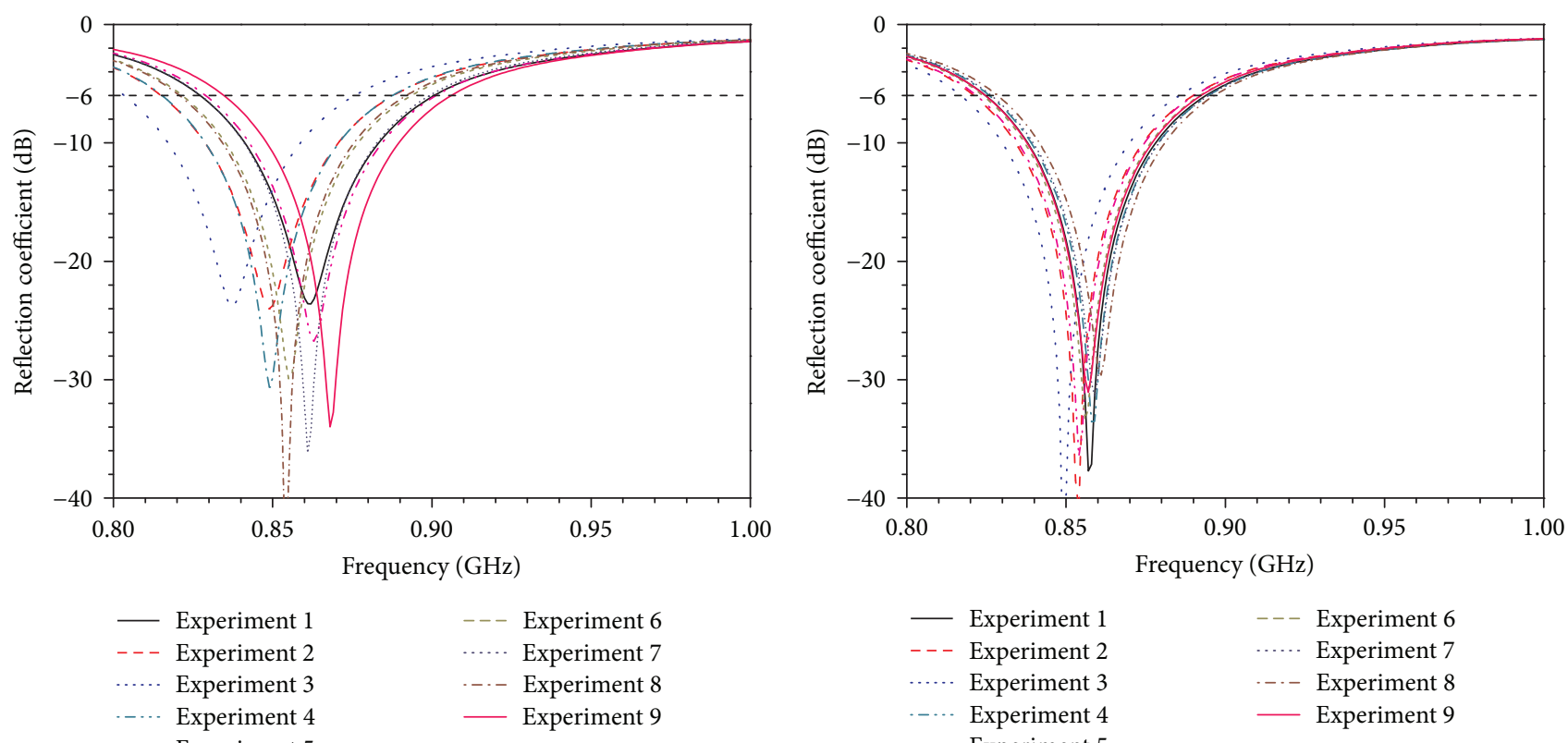

(a)

(b)

Figure 8: Simulated reflection coefficients of the antenna using the experimental parameters listed in (a) Table 3 and (b) Table 4.

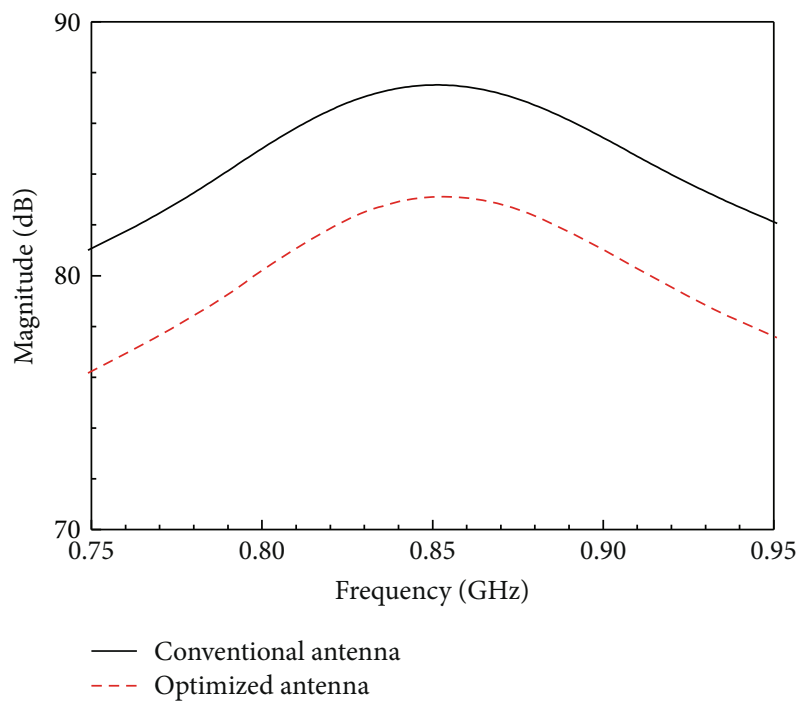

FIGURE 9: Simulated magnitudes of the electric fields at the center of $g_{2}$ in the conventional and optimized antennas.

vary from -1.56 to $0.62 \mathrm{dBi}$ and from -1.21 to $0.79 \mathrm{dBi}$, respectively. The peak gain of the optimized antenna is slightly higher than that of the conventional antenna at higher frequencies. Figure 7 shows the simulated radiation patterns for the conventional and optimized antennas. The radiation patterns are simulated at the resonant frequency of each antenna and observed on three cutting planes $(x y$-, $x z$-, and $y z$-planes). The $3 \mathrm{D}$ radiation patterns of each antenna are also depicted. The simulation results provide evidence that the radiation patterns are similar to each other.

The OA matrices for the noise factors of the conventional and optimized antennas are listed in Tables 3 and 4, respectively. Based on the design variables listed in Tables 3 and 4 , the reflection coefficients of the antennas are simulated, as shown in Figure 8. In Figure 8(a), the minimum and maximum resonant frequencies among the nine results for the conventional antenna are $837 \mathrm{MHz}$ and $868 \mathrm{MHz}$ (variance: $31 \mathrm{MHz}$ ). For the optimized antenna, the minimum and maximum resonant frequencies arise at $849 \mathrm{MHz}$ and $861 \mathrm{MHz}$ (variance: $12 \mathrm{MHz}$; see Figure $8(\mathrm{~b})$ ). These results demonstrate that the robustness of the optimized antenna is improved by $61.3 \%$ as compared to that of the conventional antenna.

\section{Experimental Result}

In this work, the metal frame exists near the IFA, which can deteriorate the robustness of the antenna. Strong undesired coupling can be generated between the metal frame and the edge of the antenna because the antenna emits most of its radiation energy from its edge. In order to investigate the degree of coupling, the magnitudes of the electric fields at the center of $g_{2}$ are simulated. These results are presented in Figure 9. The magnitudes of the electric fields in the conventional and optimized antennas are $87.5 \mathrm{~dB}$ and $83.1 \mathrm{~dB}$ at $856 \mathrm{MHz}$, respectively. This outcome indicates that the coupling between the metal frame and the antenna can be decreased by increasing $g_{2}$. For this reason, $g_{2}$ is the dominant parameter in the design of the robust antenna proposed in this paper.

To verify the experimental results, conventional and optimized antennas are fabricated and measured while varying $g_{2}$. Figure 10 shows a photograph of the fabricated models and the test jig used for the measurement. In the test jig, short and feed pins are connected to the ground plane of the PCB and SMA connectors (port), respectively. In addition, the 


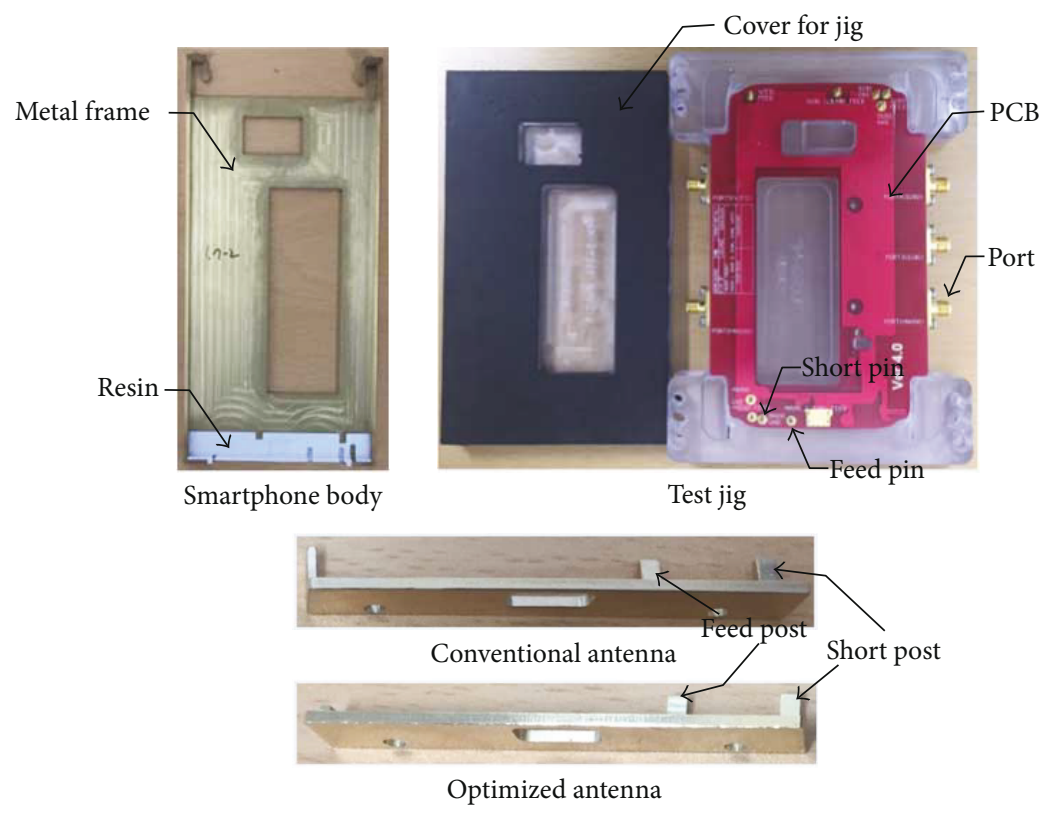

Figure 10: Photograph of the fabricated models and test jig.

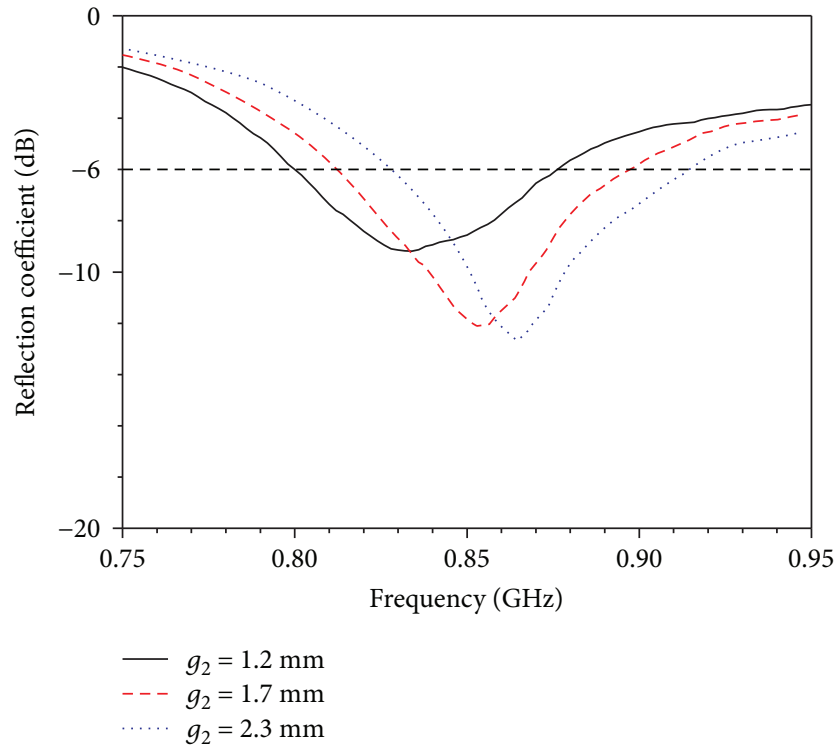

(a)

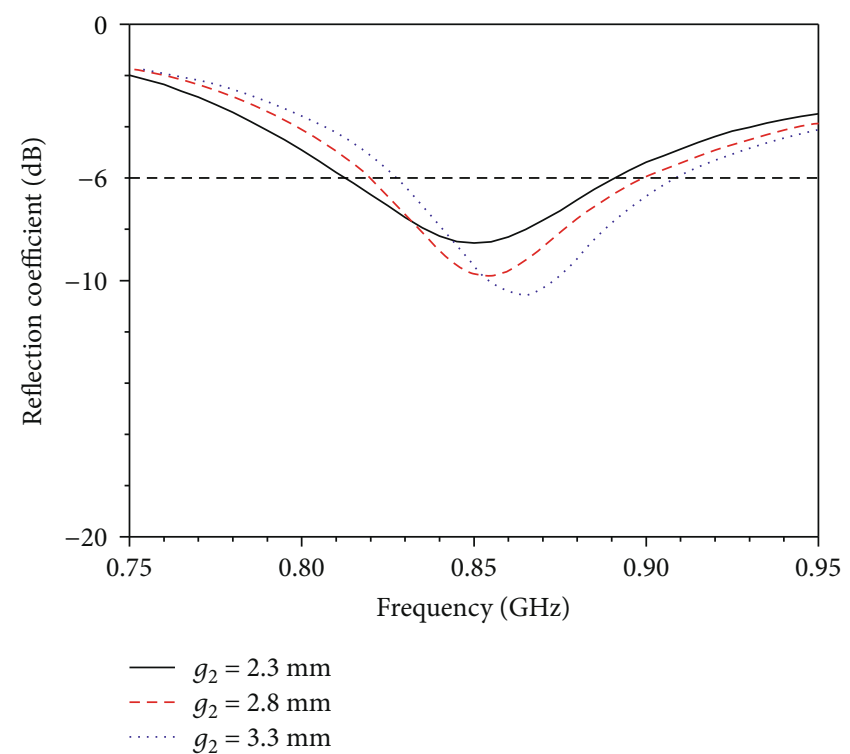

(b)

Figure 11: Measured reflection coefficients of the (a) conventional and (b) optimized antennas according to variation of the $g_{2}$.

short and feed posts of the fabricated antennas correspondingly linked to the short and feed pins on the test jig to obtain the reflection coefficient, which is measured using an Agilent 8510C network analyzer. Figure 11 shows the measured reflection coefficients of the conventional and optimized antennas according to variations of $g_{2}$. When $g_{2}$ for the conventional antenna is changed from $1.2 \mathrm{~mm}$ to $2.2 \mathrm{~mm}$, the variance of the resonant frequency is $30 \mathrm{MHz}$ $(834-864 \mathrm{MHz})$. On the other hand, for the optimized antenna, the variance is $15 \mathrm{MHz}(850-865 \mathrm{MHz})$ under identical conditions. These results indicate that the robustness of the optimized antenna is enhanced compared to that of the conventional antenna.

\section{Conclusion}

In this study, a hybrid robust optimization method developed on the basis of the GSO and OA was proposed. The proposed method was utilized to satisfy the $-6 \mathrm{~dB}$ reflection bandwidth requirement of the metal frame antenna and to derive robust design parameters for antenna manufacturing tolerances. Conventional and optimized antennas were fabricated to verify the simulated results. The simulated and measured results indicated that the robustness of the optimized antenna is enhanced by $61.3 \%$ compared to that of the conventional antenna. Therefore, the proposed optimization scheme can be feasibly 
applied to the robust design of various antennas for smartphone applications.

\section{Conflicts of Interest}

The authors declare that they have no conflicts of interest.

\section{Acknowledgments}

This work was supported by the Technical Cooperation Project of Samsung Electronics.

\section{References}

[1] H. Chen and A. Zhao, "LTE antenna design for mobile phone with metal frame," IEEE Antennas and Wireless Propagation Letters, vol. 15, pp. 1462-1465, 2016.

[2] J.-H. Ko, J.-K. Byun, J.-S. Park, and H.-S. Kim, "Robust design of dual band/polarization patch antenna using sensitivity analysis and Taguchi's method," IEEE Transactions on Magnetics, vol. 47, no. 5, pp. 1258-1261, 2011.

[3] W.-C. Weng and C. Choi, "Optimal design of CPW slot antennas using Taguchi's method," IEEE Transactions on Magnetics, vol. 45, no. 3, pp. 1542-1545, 2009.

[4] J.-H. Ko, K.-T. Kim, D.-H. Kim, H.-B. Lee, and H.-S. Kim, "A practical approach to robust design of a RFID triple-band PIFA structure," IEEE Transactions on Magnetics, vol. 46, no. 8, pp. 3333-3336, 2010.

[5] K.-T. Kim, J.-H. Ko, K. Choi, and H.-S. Kim, "Robust optimum design of PIFA for RFID mobile dongle applications," IEEE Transactions on Magnetics, vol. 47, no. 5, pp. 962-965, 2011.

[6] J.-T. Tsai, J.-H. Chou, and T.-K. Liu, "Tuning the structure and parameters of a neural network by using hybrid Taguchigenetic algorithm," IEEE Transactions on Neural Networks, vol. 17, no. 1, pp. 69-80, 2006.

[7] M. Bachlaus, M. K. Pandey, C. Mahajan, R. Shankar, and M. K. Tiwari, "Designing an integrated multi-echelon agile supply chain network: a hybrid Taguchi-particle swarm optimization approach," Journal of Intelligent Manufacturing, vol. 19, no. 6, pp. 747-761, 2008.

[8] A. Gandelli, F. Grimaccia, M. Mussetta, P. Pirinoli, and R. E. Zich, "Genetical swarm optimization: an evolutionary algorithm for antenna design," Automatika, vol. 47, no. 3-4, pp. 105-112, 2006.

[9] J. Yun and J. Choi, "Low-profile planar inverted-F antenna for ultrawideband applications," Journal of Electromagnetic Engineering and Science, vol. 16, no. 4, pp. 235-240, 2016.

[10] D. Kwon, S.-J. Lee, J.-W. Kim, B. Ahn, J.-W. Yu, and W.-S. Lee, "An eight-element compact low-profile planar MIMO antenna using LC resonance with high isolation," Journal of Electromagnetic Engineering and Science, vol. 16, no. 3, pp. 194-197, 2016.

[11] J. Yousaf, H. Jung, K. Kim, and W. Nah, "Design, analysis, and equivalent circuit modeling of dual band PIFA using a stub for performance enhancement," Journal of Electromagnetic Engineering and Science, vol. 16, no. 3, pp. 169-181, 2016.

[12] W.-C. Weng, F. Yang, and A. Z. Elsherbeni, "Linear antenna array synthesis using Taguchi's method: a novel optimization technique in electromagnetics," IEEE Transactions on Antennas and Propagation, vol. 55, no. 3, pp. 723-730, 2007. 


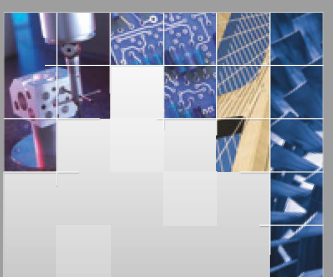

\section{Enfincering}
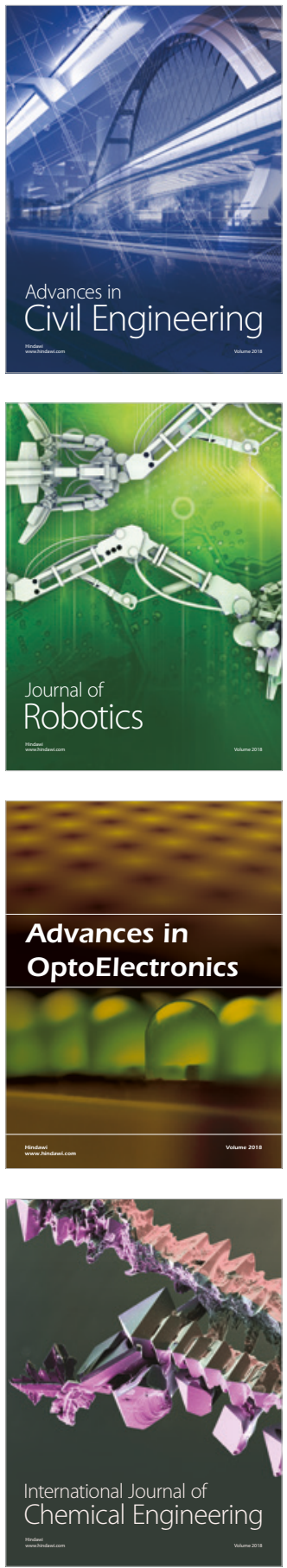

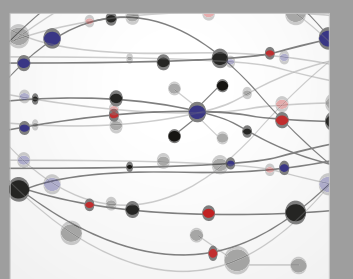

\section{Rotating \\ Machinery}

The Scientific World Journal

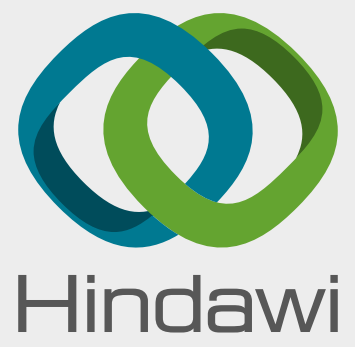

Submit your manuscripts at

www.hindawi.com
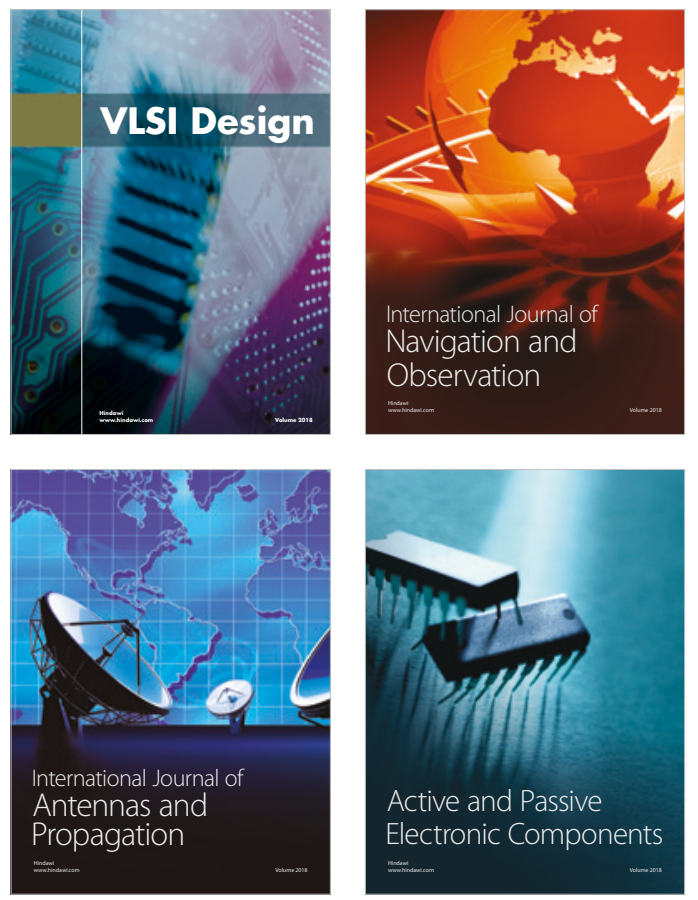
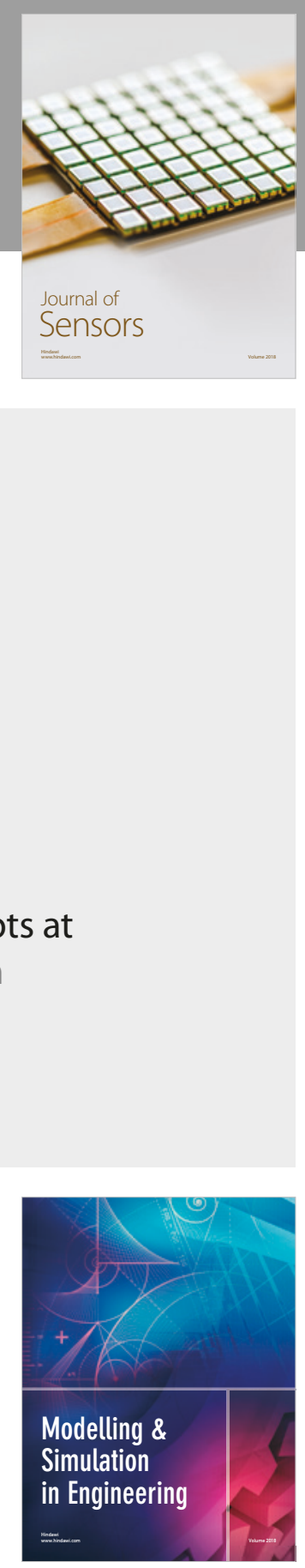

\section{Advances \\ Multimedia}
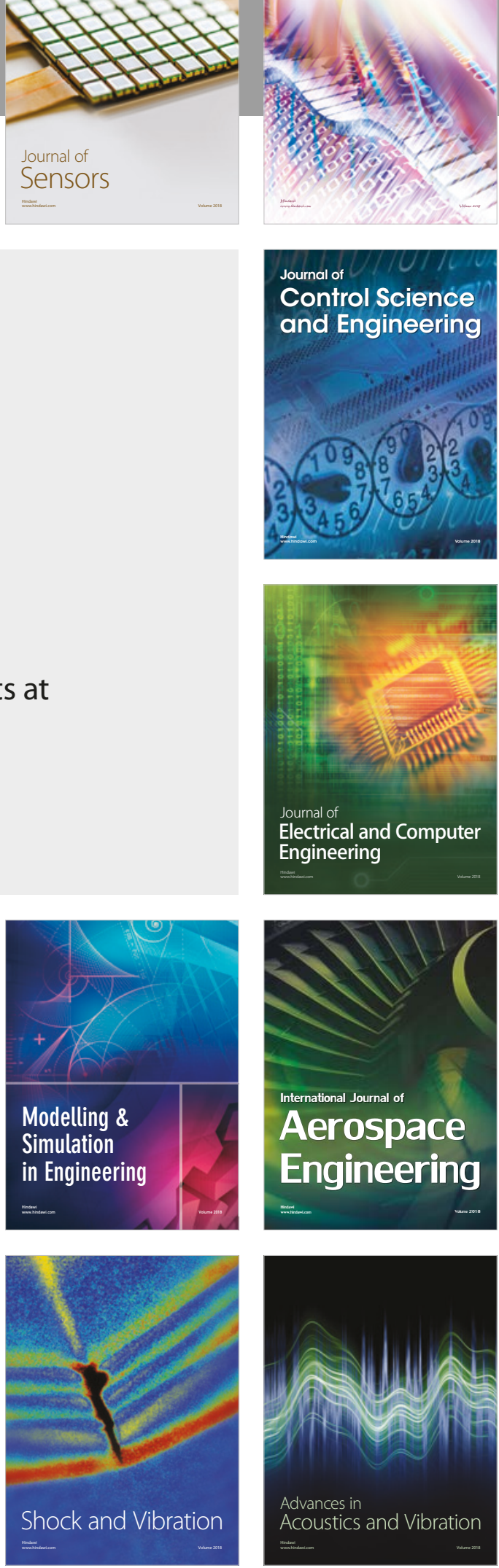